\title{
AN IOT AND BLOCKCHAIN APPROACH FOR FOOD TRACEABILITY SYSTEM IN AGRICULTURE
}

\author{
JIANLI GUO* KORHAN CENGIZ † AND RAVI TOMAR ‡
}

\begin{abstract}
The food is imperative for human being anywhere in the world and this makes the agriculture sector as the prime source of employment and need. In all the developing countries, food supply chain is the major domains of research which need a growth. The production of raw food materials is growing with advancement in technologies, but the safety of food needs urgent attention. Nowadays, the world wide serious topic is a food safety and the food safety issues are tackled by the trusted food trace-ability system. It can track and monitor the food production whole lifespan in which the processes of food raw material cultivation/breeding, processing, transporting, and selling etc. are included. This work discusses the food quality problems and further proposes the food trace-ability system based on the Internet of Things (IoT) and blockchain technique for agricultural products. The presented system architecture is detailed and other existing problems are also discussed. The consortium blockchain is utilizing as the basic network and the trace-ability system can achieve more reliable and trust able devices.
\end{abstract}

Key words: Blockchain, Food quality, IoT, Traceability system, agricultural products, Food production, Food material

AMS subject classifications. 68M25

1. Introduction. To the government, the agricultural product's quality has always been a special issue. The existence of fake and inferior commodities is reduced by the trace-ability of agricultural product realization. It is simple solution for food trace-ability in which the product information is record manually and then saved in the specific database. After that a visually query interface is provided for customers. To implement this scheme, two problems are faced that are difficult to solve. First difficulty is in the guarantee authenticity of the data recorded by the humans so there is low credibility. Second difficulty is in the monitoring of the data which is stored in centralized data centre. These all problems and difficulties can be solved by combining the IoT and the blockchain technique to the some extend. The automatic measurement and transmission is realized and making the devices intelligent by the IoT technique. Utilization of the IoT improves the data credibility and saves the lobor costs instead of manual recording. The blockchain has the various features like decentralization, Persistency, Anonymity and Auditability in the network as shown in Fig 1.1. These features make the system better as compared to the other centralized database systems. The data tampering is prevented by the blockchain technique upto the certain extend and the system become more trustable by utilizing this technique.

i. Decentralization

Based on the PP network, a distributed ledger processed and validated the set of transactions in the blockchain. The central trusted party is not required in the bitcoin blockchain and all the nodes are used for adding and verifying blockchain transactions.

ii. Persistency

Once the transaction is added to the block in the blockchain then it is not possible to or rollback an identified transaction. Moreover, invalid transactions could be discovered immediately.

iii. Anonymity

By a generated virtual identity code, there is a communication between each participant with the blockchain. Also the real identity of the participant is not uncovered. Hence, this feature raises many security challenges

\footnotetext{
${ }^{*}$ School of Computer and Software, Weifang University of Science and Technology, WQeifang, Shandong, 262700, China (jinaliguo1@gmail.com).

${ }^{\dagger}$ Department of Electrical-Electronics Engineering, Trakya University,22030, Edirne, Turkey.(korhancengiz@trakya.edu.tr).

${ }^{\ddagger}$ School of Computer Science, University of Petroleum and Energy Studies, Dehradun, India.(ravitomar7@gmail.com).
} 


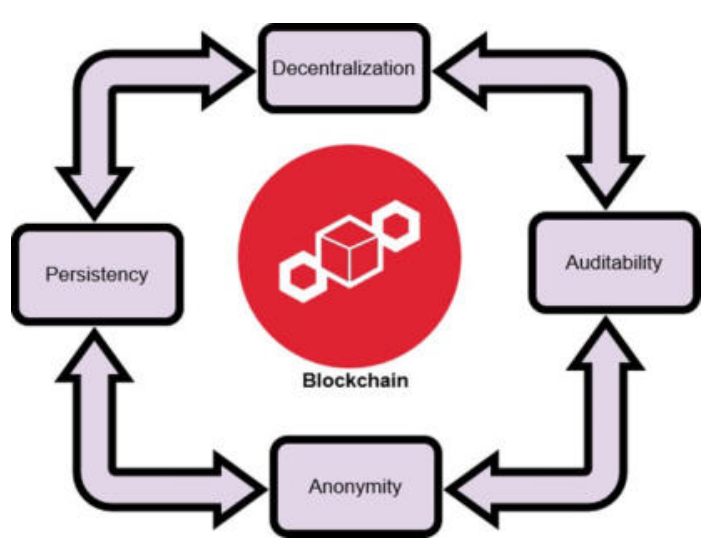

FIG. 1.1. The various features of blockchain

of blockchain transactions .

iv. Auditability Each block is linked to the last block and all the transactions and process made by the system design is verified and tracked easily.

1.1. Need for IoT in Agriculture. The surrounding environment is sensed and the necessary readings are notices by the IoT connected device which is then send to the server through the internet. All the information is stored for the future use or to the device, like a smart phone etc. where the data is viewed by the users. The system is continuously monitored and the user is allowed to take the decision on the actions to be performed. A connected network of devices is consisted by the smart sensing environment in which each other's data is constantly sent and received. In order to improve its condition, it also has the decision making capability on the user's behalf to perform the actions on environment. For further monitoring of the surroundings, this change is imposed on the environment calls and then moves continuously towards an evolving environment. In the smart system, the monitored data is sent by the user, action takes place and then actions effect on the environment. Further, the user is supported in decision making with the help of collected data.

In the agricultural food supply chain, a network of stakeholders like input supplier, Food processor makes sure that the right quantity and the quality product fetches up the right destination at the right time in the form of growing, processing or selling food to the end customer. This is all presented in the Fig 1.2.

Organization of the paper is as follows. The block-chain algorithms and related works are discussed in section 2. Section 3 details the research methodology. The experimental results are presented in section 4 and section 5 concludes the paper.

2. Related Work. The literature survey is provided in this paper for the development of the schemes utilizing block-chain technology to provide information security. A core requirement is identified and then the author proposed generalized security architecture based on block-chain. On the studied schemes, detailed cost analysis has been conducted [16].

The drawbacks in existing research are also uncovered by the comparative analysis. A model is proposed in this paper by the authors to uplift the traditional agriculture field to smart farming in which blockchain is considered with the IoT technology [17]. The equal opportunity to all stakeholders is provided by the system in the food supply chain. To reduce human interference, IoT devices are added for data collection and verification. The presented system is also compared with the scheme which only utilizes the IoT devices in the monitoring field without block-chain. As the food safety is becoming serious in the worldwide, so food safety issues are tackled the from the technical aspect and from the systems that can monitor the whole lifespan of food production [18]. The system includes the processes of food raw material cultivation/breeding, transporting, warehousing, and selling etc. The blockchain and IoT technology based open and ecological food traceability system is proposed. All parties of a smart agriculture ecosystem are involved in the system even if they may not trust each other. The manual recording and verification are replaced by the IoT devices in which human intervention is effectively reduced to the system. 


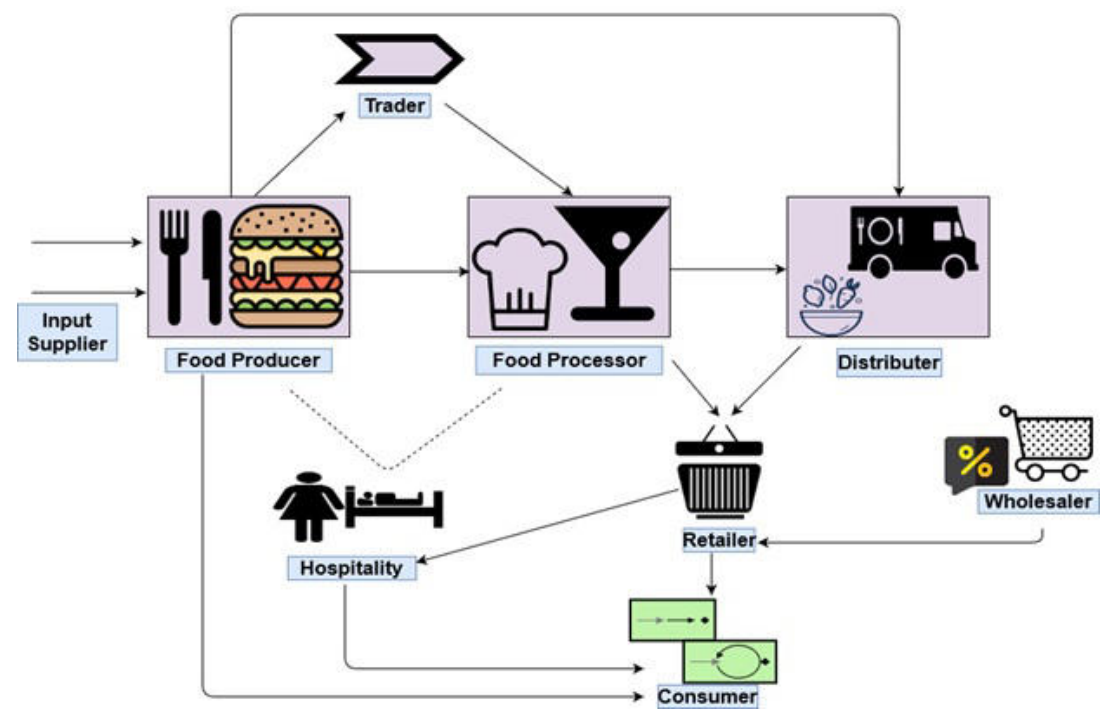

FIG. 1.2. Agricultural food supply chain

The comprehensive survey is done for the development of smart applications in precision agriculture on the importance of integrating both blockchain and IoT [19]. The novel blockchain models are proposed for major challenges in IoT-based precision agricultural systems[20]. The main functions and strengths of the common blockchain platforms are studied and reviewed for the management of various sub-sectors in precision agriculture. The security and privacy challenges are also discussed those obstacles developing blockchain-IoT systems. The application of blockchain technologies are overviewed and provided in this paper to enable the traceability in the agriculture food domain [21][22]. The levels of adoption, tools and advantages of traceability are defined in this paper[22], the functionality and advantages of blockchain technology is also overviewed briefly. The integration of block-chain and the traceability systems are also integrated and the complete review on this is provided by the author. The relevant existing commercial applications, the relevant challenges of the application of blockchain are also detailed [22].

For the interaction between people and things/objects, the IoT is a wireless network infrastructure to integrate various communication technologies. For the development of distinct applications, distinct applications for the so-called smart cities are opened by the remarkable technology. For monitoring and controlling water supply systems, IoT is used in the context of water management. The high quality water demand is increased by the climate change with the increase in the world's population and for that new water strategies are created $[23,24]$.

Block-chain is the emerging technique in which the distributed ledger is contained in the block-chain which enhances the security and data transparency [25]. In real time, blockchain is merged with the Internet of Thing. Author in this paper attempted to survey the core details of features of blockchain. The designed architecture is proposed in this paper for Smart Agriculture and ended up with some new architectural framework by merging the IOT and the BlockChain. In this paper, authors' details how the blockchain technology can help to utilized the water more effectively in the network $[26,27]$. The water ledger architecture is proposed by the author in this work. In the water management system with the ultimate goal of Water, the presented architecture serves as the basis for Blockchain helps inbuilding transparency. The block-chain technology is utilized to build the smart water management system[28-30]. For smart water management applications, IoT is the natural thing even though the different technologies essential for making it work seamlessly. An IoT-based smart water management platform is developed by the SWAMP project for precision irrigation in agriculture. The SWAMP architecture, platform is presented by the author in [31], the scalability is a major concern for IoT applications. Specially designed configurations and the re-engineering of some components are required to provide adequate performance and utilizing less computational resources [31]. 


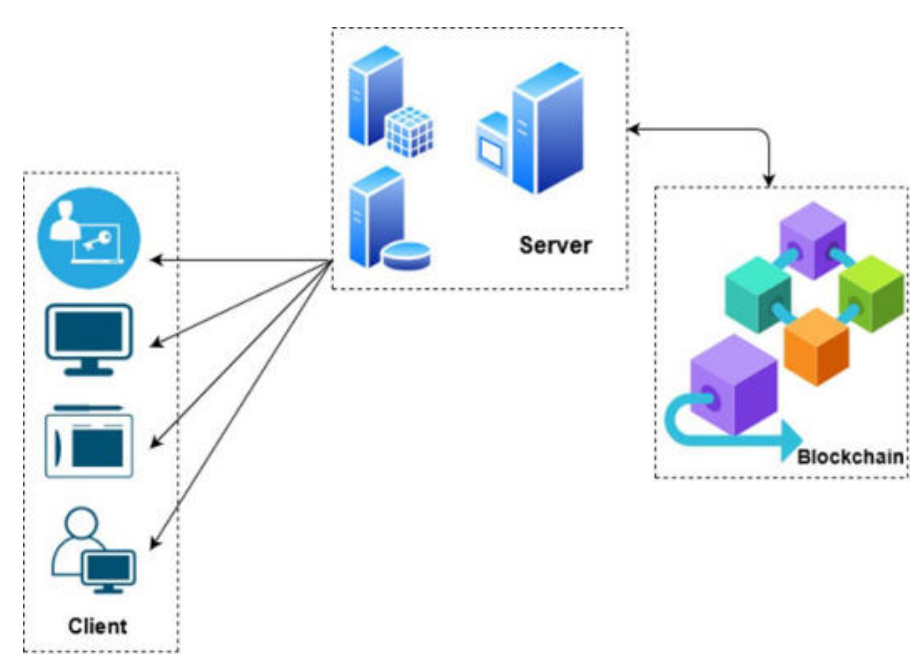

FIG. 3.1. The proposed system architecture

The impact of blockchain technology is examined by the author in the agriculture and food supply chain $[32,33]$. Existing projects, overall implications, challenges and potential are also presented and discussed with the critical view. The blockchain is the promising technology in various food products which hinder its wider popularity among farmers and systems. The IoT and blockchain technology based traceability system is presented by the author for the agriculture products in which the problems of food quality is discussed [34-36]. The system architecture and some other problems are discussed in this paper by the authors. The consortium blockchain is utilized as the basic network and the IoT devices as the recorder and achieve a more reliable, trustable and extendable traceability system.

2.1. Contribution. In this paper, food quality problems are discussed and the food traceability system is proposed which is based on the Iot and blockchain technology for agricultural products. The presented system architecture is detailed and other existing problems are also discussed. The consortium blockchain is utilizing as the basic network and the traceability system can achieve more reliable and trustable devices. There is the complexity in the agri-products supply-chain which limits the global and efficient transparency development.

3. Research Methodology. The method and the structure of the complete system is shown and detailed in this section. The proposed system structure is shown in the Figure 3.1. There are three major parts in the presented system.

i. Client

ii. Server

iii. Blockchain

Client: This part of the system has the charge of data collection. Sensors can be arranges on the farm according to the data that need to be recorded. The network module and a data processing module are included in the sensor which has the built-in processing chip. The chip in the sensor contains elliptic curve cryptographic algorithm based key pair for the block- chain network. The device identity is represented by the key pair and by registering the device; the complete data can be tracked. The manual recording interface is also provided by the system for the manual recording of the data.

Server: The system having the server is responsible for business logic processing. In this part, there are four modules in it as shown in Figure 3.2.

The employee management and IoT device management provides unified management functions for relevant devices of the enterprise. It also facilitates the operations like authorization and registration. The system parameters and security are related to the system management module.

Blockchain: The management functions are provided by this to measure the data. The function of data storage, consensus, encryption and decryption etc. are provided by the blockchain. It can increase scalabil- 


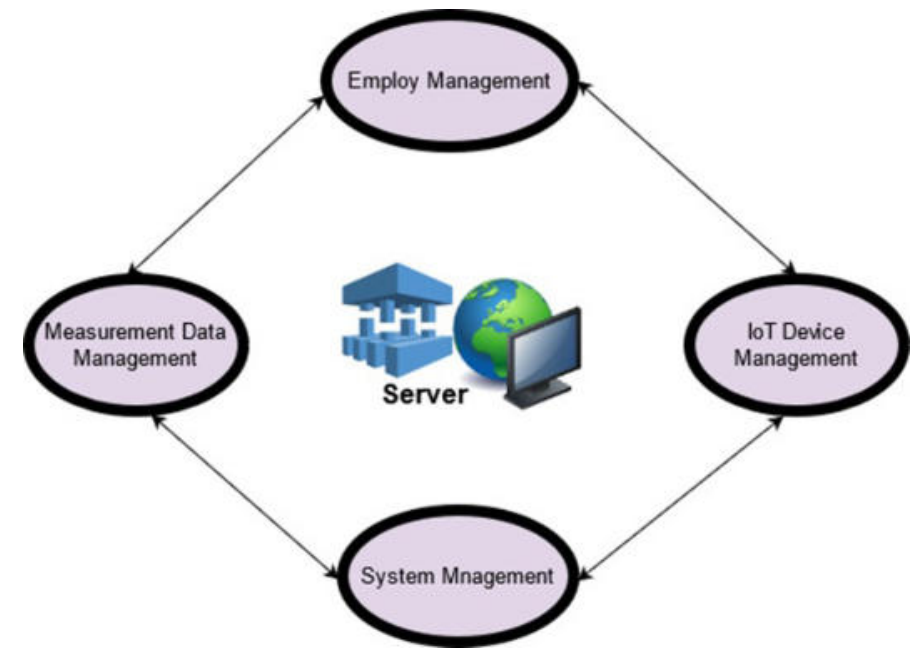

FIG. 3.2. Modules of server

ity, simplify processes and reduce costs by executing the corresponding logic at specific points in time. The blockchain base layer includes peer-to-peer networks, encryption algorithms, and membership services. The hyperledger Fabric is utilized for consortium chain building which requires joining authorization. There is communication between the nodes through peer-to-peer networks and is deployed in multiple enterprises. For the blockchain, the smart contract layer is the entry and it requires the authority verification. The core consists of the data insert and query. The user points operations are included in the core and according to the pre-defined requirements, it is set accordingly. The application layer is answerable for connecting the client and the blockchain network. The authority verification is executed by the program when it receives the request from the client. Processing results are then returned to the client. The support is provided by the extensive interface layer and facilitates access by the e-commerce systems and logistics systems etc.

3.1. Functions of IoT performed in the system. 1. The crop health is monitored by the IoT devices and the information is generated for providing support to the farmers. It supports the farmers to make a decision related to crop growth is collected and saved in the blockchain.

2. The machine learning is utilized to get the more insight information and it gives the crop related information like crop growth factor and recommendation for crop quality improvement [37, 38]. The machine learning algorithms helps the farmers to make improvement in the irrigation system.

3. The collection of high valued data will be stored by file System in decentralized server. It helps to avoid authority control and the reduce the data hacking risk. To facilitate specific stakeholders, the function of Smart contracts exchanges the data which is stored in the blockchain. It also provides the unified platform for the effective improvement.

\section{Results and Discussion.}

4.1. Experimental Analysis. The various experiments are executed and discussed here for 100 runs for each cryptographic primitive. The run time in milliseconds (maximum, minimum and average) is recorded for 100 runs and then obtained results are tabulated in the Table 4.1. Various operations are performed symbolized by the notations "Tbp, Texp, Teca, Th, Tmul and Tadd" which signifies the time required for a "bilinear pairing", a "modular exponentiation", an "elliptic curve point (scalar) multiplication", an "elliptic curve point addition", a "symmetric key encryption/decryption using the Advanced Encryption Standard". To analyse it properly, it is also shown graphically in Figure 4.1.

The proposed Scheme is evaluated and the throughput is shown graphically at different nodes for IoT implementation in agriculture sector with various nodes deployment under monitoring. Graphical representation is shown in Figure 4.2. 
TABLE 4.1

The run time in milliseconds for 100 runs

\begin{tabular}{||l|l|l||}
\hline Primitive & $\begin{array}{l}\text { Minimum Time } \\
(\mathrm{ms})\end{array}$ & $\begin{array}{l}\text { Maximum Time } \\
(\mathrm{ms})\end{array}$ \\
\hline \hline Tbp & 7.21 & 3.76 \\
\hline Texp & 0.219 & 0.062 \\
\hline Th & 0.18 & 0.01 \\
\hline Tmul & 0.006 & 0.002 \\
\hline Tadd & 0.003 & 0.001 \\
\hline Teca & 0.002 & 0.001 \\
\hline
\end{tabular}

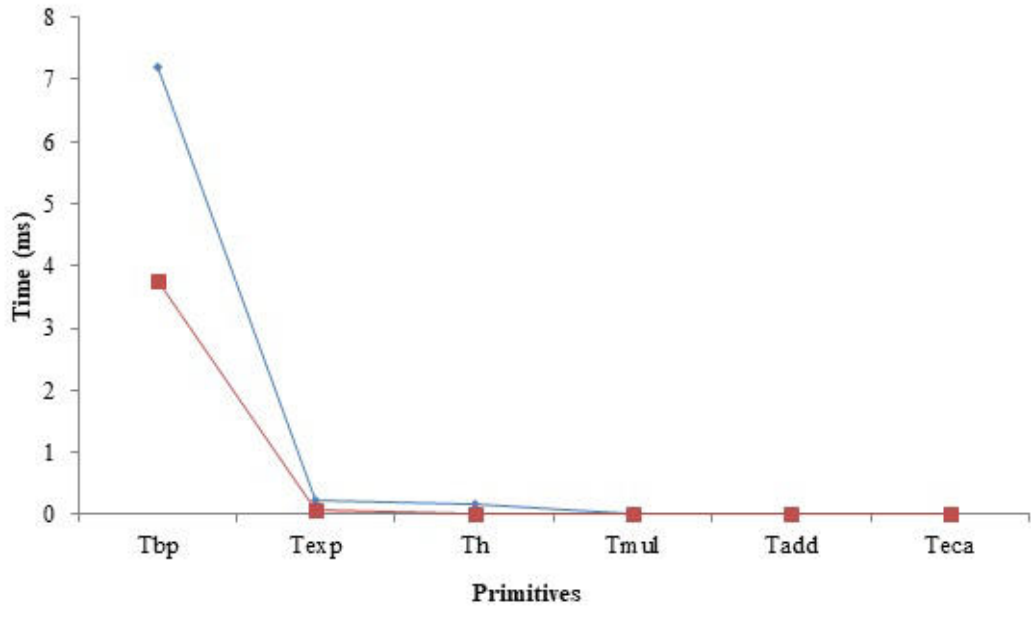

-Minimum Time (ms) - - Maximum Time (ms)

FIG. 4.1. Run time in milliseconds for 100 runs

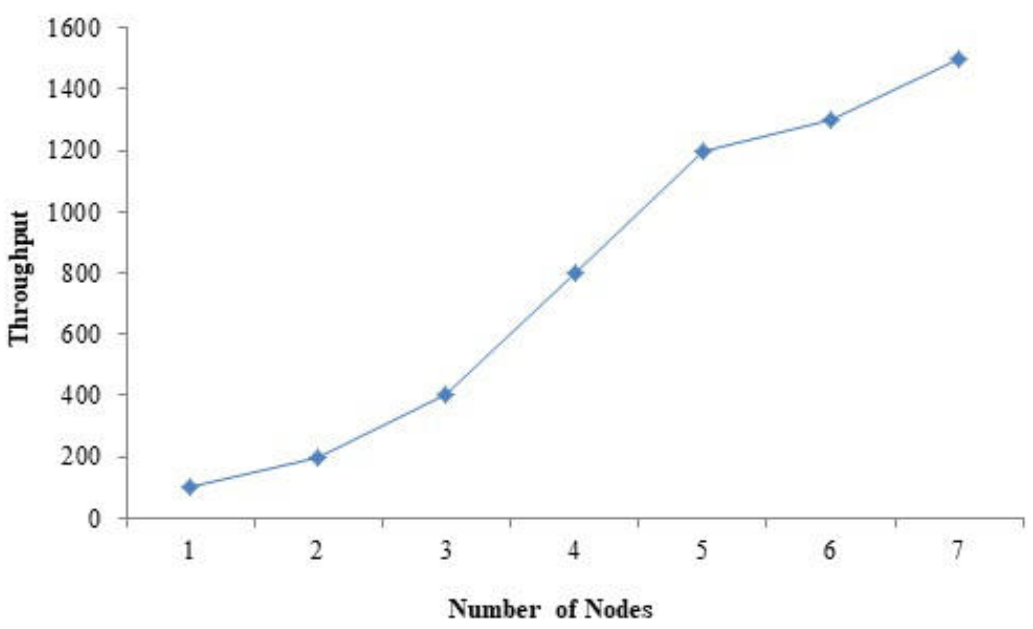

FIG. 4.2. Throughput of the proposed scheme 
TABLE 4.2

Minimum and maximum time are checked and compared with the state-of-the art technique

\begin{tabular}{|c|c|c|c|c|}
\hline Primitive & Existing $\quad$ Technique & Existing $\quad \mathrm{Te}$ & iniqueProposed Technique & Proposed Technique \\
\hline & $\begin{array}{lll}{[39]} & \text { Maximum } & \text { Time } \\
(\mathrm{ms}) & & \end{array}$ & {$\left[\begin{array}{ll}{[39]} & \text { Minimum } \\
(\mathrm{ms}) & \end{array}\right.$} & TimeMaximum Time (ms) & Minimum Time (ms) \\
\hline Tbp & 8.440 & 4.424 & 7.210 & 3.763 \\
\hline Texp & 0.241 & 0.043 & 0.219 & 0.062 \\
\hline $\mathrm{Th}$ & 0.143 & 0.026 & 0.184 & 0.014 \\
\hline Tmul & 0.007 & 0.001 & 0.006 & 0.001 \\
\hline Tadd & 0.003 & 0.001 & 0.003 & 0.001 \\
\hline Teca & 0.002 & 0.001 & 0.002 & 0.001 \\
\hline
\end{tabular}

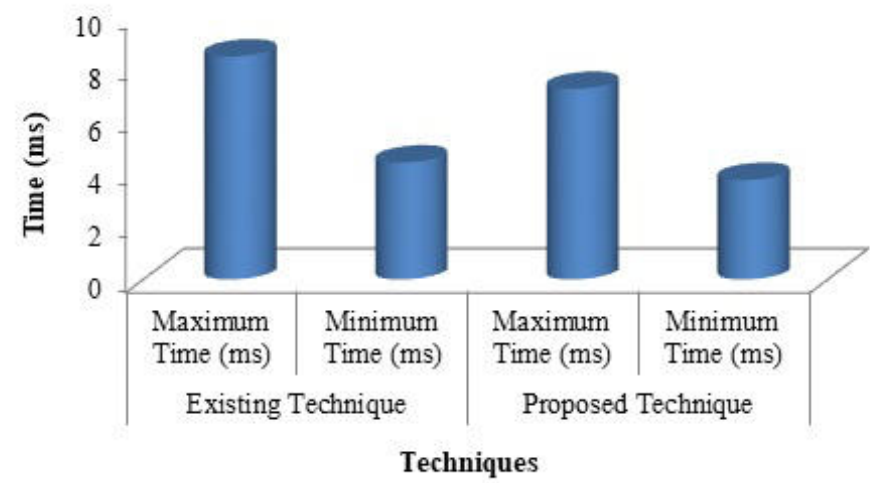

FIG. 4.3. Time for Tbp

With the blockchain and IOT integration, the presented scheme shows the improvement and the obtained results shows a tremendous raise from the IoT based scheme. The proposed scheme is validated in contrast to the various nodes which are tested in the agriculture. It is also seen that the throughput of the proposed scheme is higher as compared to the other existing schemes. With the emphasis on the different types of blockchains, the working of blockchain system and their applications in the various domains is discussed.

4.2. The proposed technique comparison with the existing techniques. The comparison of the results obtained by the presented technique is done with the state-of-the-art technique for the validation purpose. This comparison shows the improvement of the proposed technique. The minimum and maximum time are checked and compared with the state-of-the art technique for different primitives like Tbp, Texp, Teca, Th, Tmul, Tadd, Tecsiggen and Tecsigver which signifies the time required for a "bilinear pairing", a "modular exponentiation", an "elliptic curve point (scalar) multiplication", an "elliptic curve point addition", a "symmetric key encryption/decryption as tabulated in Table 4.2.

The minimum and maximum time obtained for the different primitives by the proposed and the existing technique is also shown graphically in Fig 4.3- Fig 4.8. The graphical representation gives the better analytic details and the better visualization.

The maximum time required by the proposed techniques for different primitives is less as compared to the existing technique and the minimum times requirement by both the techniques are comparable with less complexity of the presented technique. The percentage improvement of the proposed technique is also calculated and shown in Fig 4.9. It shows the overall performance improvement of the proposed technique over the existing technique.

The Figure 4.9 shows that the presented technique is $23.74 \%$ and $18.92 \%$ better than the existing technique 


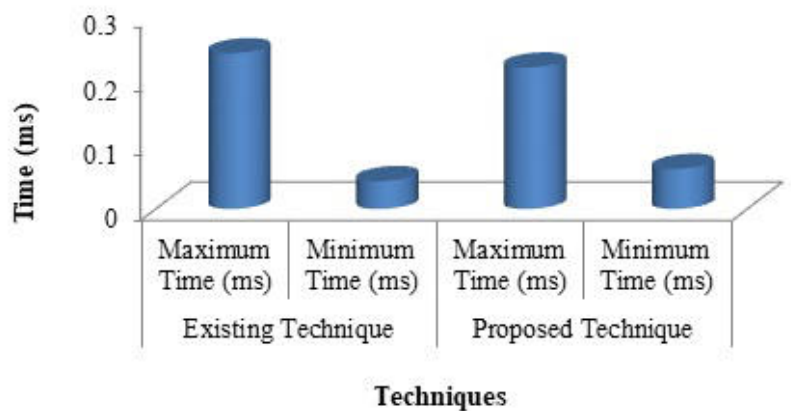

FIG. 4.4. Time for Texp

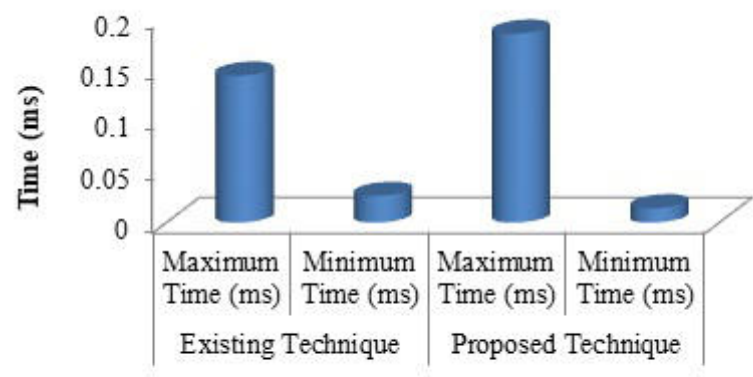

Techniques

FIG. 4.5. Time for Th

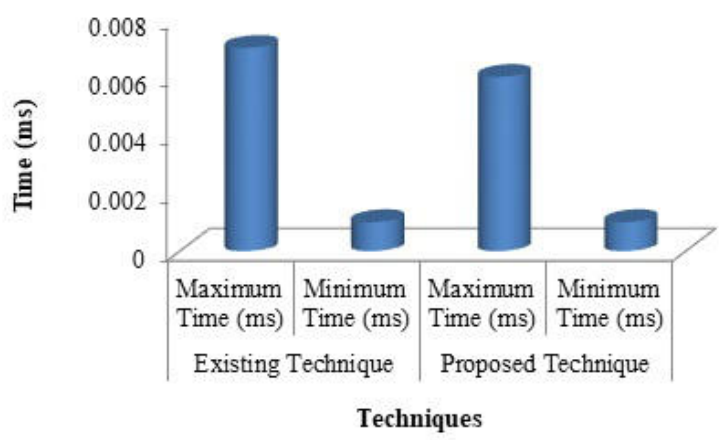

Fig. 4.6. Time for Tmul

in terms of maximum and minimum time (ms). It outperforms the existing techniques in terms of time interval for different primitives. The presented technique is very advantages; also there are many challenges in the blockchain technique.

1. Scalability: The issue in the blockchain is the storage space that if the blocks added in the blockchain then it cannot be deleted. The number of transactions processed per unit time is limited and in the small and 


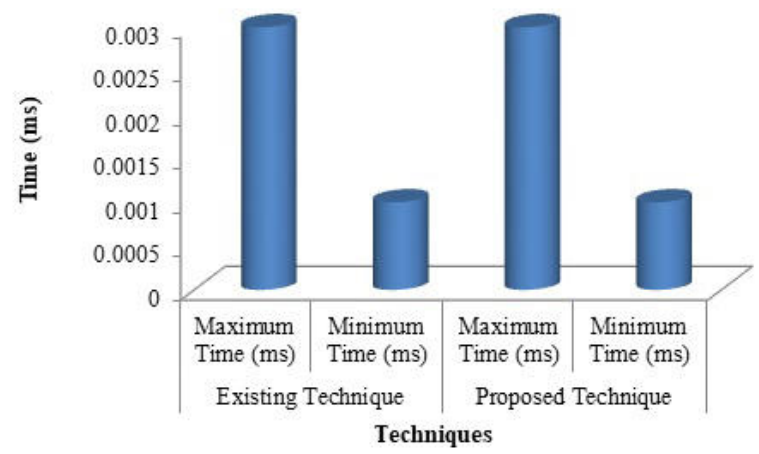

FIG. 4.7. Time for Tadd

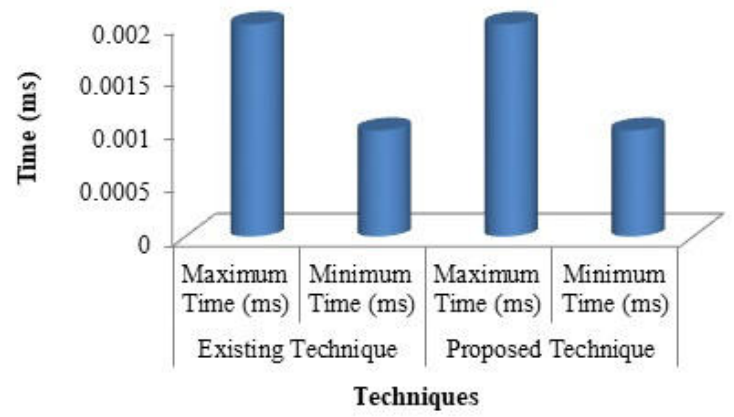

FIG. 4.8. Time for Teca

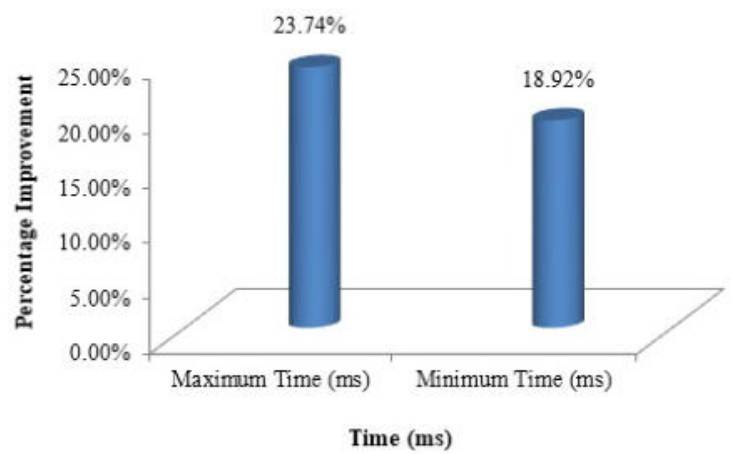

FIG. 4.9. Percentage improvement of the proposed technique

large blocks, there is always a tradeoff.

2. Privacy Leakage: The transaction contents are always available publically, hence the transactional privacy is not ensured by the blockchain.

3. Selfish Mining: If the some of the nodes of blockchain are not honest then the blockchains are susceptible to collusion.

5. Conclusion. The companies of food supply chain find the actual food source and the food production whole process is tracked from origin to end consumer. In this paper, food quality problems are discussed and the food traceability system is proposed which is based on the IoT and blockchain technology for agricultural 
products. The presented system architecture is detailed and other existing problems are also discussed. The consortium block-chain is utilizing as the basic network and the traceability system can achieve more reliable and trustable devices. Utilization of the IoT improves the data credibility and saves the lobor costs instead of manual recording. With the integration of blockchain with the IoT, the presented scheme shows the improvement and the obtained results shows a tremendous raise from the IoT based techniques. The hybrid technique can be designed for better and efficient results in the future.

\section{REFERENCES}

[1] Srilakshmi, A., Rakkini, J., Sekar, K. R. and Manikandan, R., A comparative study on Internet of Things (IoT) and its applications in smart agriculture, Pharmacognosy Journal, 10(2), 2018.

[2] Kokkonis, G., Kontogiannis, S.and Tomtsis, D. , A smart Iot fuzzy irrigation system, Power (mW), $100(63), 25,2017$.

[3] McCready, M. S., Dukes, M. D. And Miller, G. L., Water conservation potential of smart irrigation controllers on St. Augustinegrass, Agricultural water management, 96(11), 1623-1632, 2009.

[4] Du, M., Chen, Q., XIAO, J., Yang, H. And Ma, X. , Supply chain finance innovation using blockchain, IEEE Transactions on Engineering Management, 67(4), 1045-1058, 2020.

[5] Caro, M. P., Ali, M. S., Vecchio, M. and Giaffreda, R. , Blockchain-based traceability in Agri-Food supply chain management: A practical implementation, In 2018 IoT Vertical and Topical Summit on Agriculture-Tuscany (IOT Tuscany) (pp. 1-4). IEEE, 2018.

[6] Tian, F. , An agri-food supply chain traceability system for China based on RFID and blockchain technology, In 2016 13th international conference on service systems and service management (ICSSSM) (pp. 1-6). IEEE, 2016.

[7] Galvez, J. F., Mejuto, J. C. and Simal-Gandara, J., Future challenges on the use of blockchain for food traceability analysis, TrAC Trends in Analytical Chemistry, 107, 222-232, 2018.

[8] Zhao, G., Liu, S., Lopez, C., Lu, H., Elgueta, S., Chen, H., And Boshkoska, B. M. , Blockchain technology in agri-food value chain management: A synthesis of applications, challenges and future research directions, Computers in Industry, 109, 83-99, 2019.

[9] Kumar, M. V., And Iyengar, N. C. S. , A framework for Blockchain technology in rice supply chain management, Adv. Sci. Technol. Lett, 146, 125-13, 2017.

[10] LI, J., WANG, X., Research on the application of blockchain in the traceability system of agricultural products, In $20182 \mathrm{nd}$ IEEE Advanced Information Management, Communicates, Electronic and Automation Control Conference (IMCEC) (pp. 2637-2640). IEEE, 2018.

[11] Colombo, P. E., Patterson, E., Elinder, L. S., Lindroos, A. K., Sonesson, U., Darmon, N. and Parlesak, A. , Optimizing school food supply: integrating environmental, health, economic, and cultural dimensions of diet sustainability with linear programming, International Journal of Environmental Research and Public Health, 16(17), 2019.

[12] Kamilaris, A., Fonts, A. and Prenafeta-Bold, F. X. , The rise of blockchain technology in agriculture and food supply chains, Trends in Food Science and Technology, 91, 640-652, 2019.

[13] Wood, A. D., Stankovic, J. A. , Denial of service in sensor networks, computer, 35(10), 54-62, 2002.

[14] Monrat, A. A., Schelén, O., And Andersson, K. , A survey of blockchain from the perspectives of applications, challenges, and opportunities, IEEE Access, 7, 117134-117151, 2019.

[15] Mekala, M. S. and Viswanathan, P. , A Survey: Smart agriculture IoT with cloud computing, In 2017 international conference on microelectronic devices, circuits and systems (ICMDCS) (pp. 1-7). IEEE.

[16] Akmandor, A. O., Hongxu, Y. I. N. And Jha, N. K. , Smart, secure, yet energy-efficient, Internet-of-Things sensors, IEEE Transactions on Multi-Scale Computing Systems, 4(4), 914-930, 2018.

[17] Awan, S. H., Ahmed, S., Safwan, N., Najam, Z., Hashim, M. Z.and Safdar, T. , Role of Internet of Things (IoT) with blockchain technology for the development of smart farming. J. Mech, Continua Math. Sci., 14(5), 170-188, 2019.

[18] Tsang, Y. P., Choy, K. L., Wu, C. H., Ho, G. T. S. and Lam, H. Y. , Blockchain-driven IoT for food traceability with an integrated consensus mechanism, IEEE access, 7, 129000-129017, 2019.

[19] Ahmed, N., De, D. And Hussain, I. , Internet of Things (IoT) for smart precision agriculture and farming in rural areas, IEEE Internet of Things Journal, 5(6), 4890-4899, 2018.

[20] M ToRkya, A E HassaneInB, Integrating blockchain and the internet of things in precision agriculture: Analysis, opportunities, and challenges, Computers and Electronics in Agriculture, pp 1-23, 178, 2020

[21] Demestichas, K., Peppes, N., Alexakis, T. and Adamopoulou, E. , Blockchain in Agriculture Traceability Systems: A Review, Applied Sciences, 10(12), 4113, 2020.

[22] A. Vangala, A. K. Das, N. Kumar and M. Alazab,Smart Secure Sensing for IoT-Based Agriculture: Blockchain Perspective, IEEE Sensors Journal, 21(16), pp. 17591-17607, 2021.

[23] Rajakumar, G., Sankari, M. S., Shunmugapriya, D.and Maheswari, S. U. , Iot based smart agricultural monitoring system, Asian J. Appl. Sci. Technol, 2, 474-480, 2018.

[24] Khoa, T. A., Man, M. M., Nguyen, T. Y., Nguyen, V. and Nam, N. H. , Smart Agriculture Using IoT Multi-Sensors: A Novel Watering Management System, Journal of Sensor and Actuator Networks, 8(3), 45, 2019.

[25] Devi, M. S., Suguna, R., Joshi, A. S. and Bagate, R. A. , Design of IoT blockchain based smart agriculture for enlightening safety and security, In International Conference on Emerging Technologies in Computer Engineering (pp. 7-19). Springer, Singapore, 2019. 
[26] Kumar, M. V. and Iyengar, N. C. S. , A framework for Blockchain technology in rice supply chain management, Adv. Sci. Technol. Lett, 146, 125-130, 2019.

[27] Kamienski, C., Soininen, J. P., Taumberger, M., Dantas, R., Toscano, A., Salmon Cinotti, T.and Torre Neto, A. , Smart water management platform: Iot-based precision irrigation for agriculture, Sensors, 19(2), 276, 2019.

[28] García, L., Parra, L., Jimenez, J. M., Lloret, J. and Lorenz, P. , IoT-Based Smart Irrigation Systems: An Overview on the Recent Trends on Sensors and IoT Systems for Irrigation in Precision Agriculture, Sensors, 20(4), 1042, 2020.

[29] Kamilaris, A., Fonts, A. and Prenafeta-Bold, F. X. , The rise of blockchain technology in agriculture and food supply chains, Trends in Food Science and Technology, 91, 640-652,2019.

[30] Ruan, J., Wang, Y., Chan, F. T. S., Hu, X., ZhaO, M., Zhu, F. And Lin, F. , A life cycle framework of green IoT-based agriculture and its finance, operation and management issues, IEEE communications magazine, 57(3), 90-96,2019.

[31] Kamienski, C.; Soininen, J.-P.; Taumberger, M.; Dantas, R.; Toscano, A.; Salmon Cinotti, T.; Filev Maia, R.; Torre Neto, A. Smart Water Management Platform: IoT-Based Precision Irrigation for Agriculture, Sensors, 19(276), 2019.

[32] Rathee, G., Sharma, A., Saini, H., Kumar, R. and Iqbal, R., A hybrid framework for multimedia data processing in IoT-healthcare using blockchain technology, Multimedia Tools and Applications, 1-23,2019.

[33] Rathee, G., Sharma, A., Iqbal, R., Aloqaily, M., Jaglan, N. and Kumar, R. , A blockchain framework for securing connected and autonomous vehicles, Sensors, 19(14), 3165,2019.

[34] Kokkonis, G., Kontogiannis, S. And Tomtsis, D. , A smart Io T fuzzy irrigation system, Power (mW), $100(63), 25,2017$.

[35] Atram, H. F., Alenezi, A., Alassafi, M. O. and Wills, G. , Blockchain with internet of things: Benefits, challenges, and future directions, International Journal of Intelligent Systems and Applications, 10(6), 40-48,2018.

[36] Xia, F., YAng, L. T., WAng, L. And Vinel, A. , Internet of things, International journal of communication systems, 25(9), $1101,2012$.

[37] Shackelford, S. J. And Myers, S., Block-by-block: leveraging the power of blockchain technology to build trust and promote cyber peace, Yale JL and Tech., 19, 334, 2017.

[38] Sharma, A., Tomar, R., Chilamkurti, N. and Kim, B. G. , Blockchain based smart contracts for internet of medical things in e-healthcare, Electronics, 9(10), 1609, 2020.

[39] Li, W., Song, H. and Zeng, F. , Policy-based secure and trustworthy sensing for internet of things in smart cities, IEEE Internet of Things Journal, 5(2), 716-723, 2017.

Edited by: Pradeep Kumar Singh et al.

Received: May 13, 2021

Accepted: Sep 20, 2021 